\title{
Geometrical Approach for Grasp Synthesis on Discretized 3D Objects*
}

\author{
Máximo A. Roa and Raúl Suárez \\ Institute of Industrial and Control Engineering (IOC) \\ Technical University of Catalonia (UPC), Barcelona, Spain \\ Emails:maximo.roa@upc.edu,raul.suarez@upc.edu
}

\begin{abstract}
Grasp synthesis on real 3D objects is a critical problem in grasp and manipulation planning. This paper presents a geometrical approach to compute force closure (FC) grasps, with or without friction and with any number of fingers. The object's surface is discretized in a cloud of points, so the algorithm is applicable to objects of any arbitrary shape. One or more FC grasps are obtained with a geometrical approach, which embeds the FC test in the algorithm to simplify achieving the force-closure property. This initial FC grasp may be improved with a complementary optimization algorithm. The grasp quality is measured considering the largest perturbation wrench that the grasp can resist with independence of the perturbation direction. The efficiency of both algorithms is illustrated through numerical examples.
\end{abstract}

Index Terms-Grasp planning, force-closure grasps.

\section{INTRODUCTION}

Grasps capable of ensuring the immobility of the object under external disturbances satisfy one of the following properties: form-closure, when it is the positions of the fingers that guarantee the object immobility, or force-closure, when it is the forces applied by the fingers that guarantee the object immobility [1]. Based on any of these properties, the grasp planners calculate the position of the fingers on the object surface. The property to be used largely depends on the field of application: form-closure is used when the task requires a robust grasp not relying on friction, e.g. the fixture of objects to be manufactured or inspected, while force-closure is specially used in grasping and manipulation of objects with a lower number of frictional contacts using for instance mechanical grippers or hands. Several algorithms have been developed to determine grasps with different number of fingers and satisfying the form or forceclosure condition in 2D polygonal [2] or non-polygonal [3] objects, 3D polyhedral objects [4] or objects with smooth curved surfaces [5]. However, the development of algorithms to efficiently synthesize grasps in 3D complex real-world objects is still an open research problem.

A widely used technique to represent an arbitrary object is the approximation of the object surface with a triangular mesh with a high number of faces, or equivalently, with

*This work was partially supported by the CICYT projects DPI2004-03104 and DPI2005-00112, and Acción Integrada HI2005-0290

Máximo Roa works at the National University of Colombia. He is supported by $\mathrm{Al} \beta \mathrm{an}$, the European Union Programme of High Level Scholarships for Latin America, scholarship No.E04D039103CO a set of surface points and their corresponding normal direction [6], provided that the number of triangles or points is large enough to accurately represent the surface. The application of current algorithms developed for grasp synthesis of polyhedral objects to this kind of object representations would have a large computational cost, so new algorithms are being developed. A strategy based on random generation of grasps was shown to be quick and efficient to generate good grasps on arbitrary objects [7]; the complexity of such grasp planner depends on the geometric form of the object (not on its number of faces), but the generated grasps are not optimal. Wang [8] proposes an algorithm for fixture synthesis on discrete objects minimizing the workpiece positioning errors due to uncertainties in the position of the locators and in the geometry of the workpiece. Ding et al. [9] propose an algorithm to generate a form-closure grasp with seven frictionless contact points; however, it can be trapped in local minima. Liu et al. [10] extend the previous algorithm to find one force-closure grasp with frictional or frictionless contact points, and Niparnan and Sudsang [11] generate several 4-finger concurrent force-closure grasps; their contribution and the comparison with the algorithms presented in this paper will be discussed later.

This paper deals with the problem of finding a forceclosure (FC) grasp with frictional or frictionless contact points, and with any number $n$ of contacts, provided that $n \geq 3$ for frictional grasps and $n \geq 7$ for frictionless grasps. The proposed approach comprises two algorithms. The first algorithm finds at least one FC grasp with a geometrical procedure that avoids a costly FC test in each iteration. The second algorithm optimizes an initial FC grasp to get a locally optimum grasp; the optimization procedure looks for the grasp with the largest resisted perturbation wrench, with independence of the perturbation direction [12].

The rest of the paper is structured as follows. Section II provides an overview of the problem, including the main assumptions in the object and contact models, and discusses the used FC test and grasp quality measure. Section III presents the algorithm to compute at least one FC grasp. Section III provides an additional algorithm to compute locally optimum FC grasps with any number of fingers. Both algorithms have been implemented and Section $\mathrm{V}$ shows the results of their application to several objects. Finally, Section VI presents the conclusions of the work. 


\section{PRoblem OVERVIEW}

\section{A. Problem definition}

The problem to be tackled is the search of a FC grasp in a set of points representing the surface of an arbitrary 3D object. The work relies on the following assumptions:

- The object surface is represented with a large set $\Omega$ of points, described by position vectors $\boldsymbol{p}_{i}$ measured with respect to a reference system located in the object's center of mass $(C M)$. Each point has an associated normal direction $\hat{\boldsymbol{n}}_{i}$ pointing towards the interior of the object.

- The number of points in $\Omega$ is large enough to accurately represent the surface of the object.

This paper proposes a geometric approach to synthesize appropriate FC grasps, with two main algorithms:

1) An algorithm to search the set of points $\Omega$ for one or several FC grasps, regardless of its quality.

2) An optimization algorithm that searches for a local optimum grasp, measured with the largest perturbation wrench that the grasp can resist, and starting from one FC grasp obtained, for instance, with the previous algorithm.

\section{B. Frictionless grasps}

Seven frictionless contacts are necessary and sufficient to hold a 3D object with a FC grasp, provided that the object has no rotational symmetries [13]. With frictionless contact points, the grasp forces can only be applied in the direction normal to the object surface. A force $\boldsymbol{f}_{i}=\alpha_{i} \hat{\boldsymbol{n}}_{i}$ applied on the object at the point $\boldsymbol{p}_{i}$ generates a torque $\boldsymbol{\tau}_{i}=\boldsymbol{p}_{i} \times \boldsymbol{f}_{i}$ with respect to $C M$, with $\alpha_{i}$ being a nonnegative value representing the magnitude of the grasping force. The force and the torque are grouped together in a wrench vector (also known as generalized force vector) given by

$$
\tilde{\boldsymbol{\omega}}_{i}=\left(\begin{array}{c}
\boldsymbol{f}_{i} \\
\boldsymbol{\tau}_{i}
\end{array}\right)=\alpha_{i}\left(\begin{array}{c}
\hat{\boldsymbol{n}}_{i} \\
\boldsymbol{p}_{i} \times \hat{\boldsymbol{n}}_{i}
\end{array}\right)
$$

The wrenches applied through the contact points on the object can be grouped in a wrench set $W=\left\{\boldsymbol{\omega}_{1}, \boldsymbol{\omega}_{2}, \ldots, \boldsymbol{\omega}_{7}\right\}$, where each $\boldsymbol{\omega}_{i}, i=1, \ldots, 7$, is called a primitive contact wrench when $\alpha_{i}=1$ in equation (1). Each physical point $\boldsymbol{p}_{i}$ in the set $\Omega$ has a corresponding wrench $\boldsymbol{\omega}_{i}$ in the generalized force space; both of them will be used to indicate a grasp point.

\section{Frictional grasps}

Coulomb's friction model is used in this work, stating that there is no slipping at the contact point if $\boldsymbol{f}_{i}^{t} \leq \mu \boldsymbol{f}_{i}^{n}$, with $\mu$ being the friction coefficient. In the three-dimensional physical space this is a nonlinear model, defining a friction cone that includes all the possible grasp forces. To simplify the model, the cone is linearized with a $m$-side polyhedral convex cone (the more sides the better the approximation, but the greater the computational cost to deal with the linearized cone).

The grasping force at the contact point is given by

$$
\boldsymbol{f}_{i}=\sum_{j=1}^{m} \alpha_{i j} \boldsymbol{s}_{i j}, \quad \alpha_{i j} \geq 0
$$

with $s_{i j}$ representing the normalized vector of the $j$-th edge of the convex cone. The wrench produced by the force $\boldsymbol{f}_{i}$ is

$$
\tilde{\boldsymbol{\omega}}_{\boldsymbol{i}}=\sum_{j=1}^{m} \alpha_{i j} \boldsymbol{\omega}_{i j}, \quad \boldsymbol{\omega}_{i j}=\left(\begin{array}{c}
\boldsymbol{s}_{\boldsymbol{i j}} \\
\boldsymbol{p}_{\boldsymbol{i}} \times \boldsymbol{s}_{\boldsymbol{i} \boldsymbol{j}}
\end{array}\right)
$$

where $\boldsymbol{\omega}_{i j}$ are the primitive contact wrenches in the frictional case. Therefore, each contact point has $m$ associated points in the wrench space, one for each side $s_{i j}$ of the pyramid. Let $\boldsymbol{\omega}_{i}$ be the "normal contact wrench" for the force $\boldsymbol{f}_{i}$, i.e. the primitive contact wrench in case of a frictionless contact point. The following relation can be established between the normal contact wrench $\boldsymbol{\omega}_{i}$ and the primitive contact wrenches $\boldsymbol{\omega}_{i j}$ for the linearized friction cone in a particular contact point:

$$
\boldsymbol{\omega}_{\boldsymbol{i}}=\frac{1}{m} \sum_{j=1}^{m} \boldsymbol{\omega}_{\boldsymbol{i} j}
$$

For a given grasp $G=\left\{\boldsymbol{p}_{1}, \ldots, \boldsymbol{p}_{n}\right\}$ (or equivalently, $\left.G=\left\{\boldsymbol{\omega}_{1}, \ldots, \boldsymbol{\omega}_{n}\right\}\right)$, the corresponding wrench set is $W=\left\{\boldsymbol{\omega}_{11}, \ldots, \boldsymbol{\omega}_{1 m}, \ldots, \boldsymbol{\omega}_{n 1}, \ldots, \boldsymbol{\omega}_{n m}\right\}$.

\section{Force-closure test}

Several criteria have been proposed to test the forceclosure property in a particular grasp. A necessary and sufficient condition for the existence of a FC grasp is that the origin of the wrench space lies strictly inside the convex hull $(\mathrm{CH})$ of the primitive contact wrenches [14]. This condition can be solved as a linear programming problem using a rayshooting technique [15], or as a particular application of an algorithm to find the distance between a point and a polytope [16]. The FC test used in this work is based on the following lemma.

Lemma 1: Let $G$ be a grasp with a set $W$ of primitive contact wrenches, $\mathcal{I}$ the set of strictly interior points of $C H(W)$, and $H$ a supporting hyperplane of $C H(W)$ (i.e. a hyperplane containing one of the facets of $C H(W)$ ). The origin of the wrench space $O \in \mathcal{I}$ if and only if any $P \in \mathcal{I}$ and $O$ are in the same half-space for every $H$ of $C H(W)$.

From Lemma 1, checking whether a given point $P \in \mathcal{I}$ and the origin $O$ lie in the same half-space defined by every supporting hyperplane $H$ of $C H(W)$ is enough to prove whether $O$ lies inside $C H(W)$, i.e. to prove the FC property for the grasp $G . P$ is chosen as the centroid of the primitive contact wrenches, which is always an interior point of $C H(W)$. Then, the FC test verifies if the centroid $P$ and the origin $O$ lie on the same side for all the supporting hyperplanes of $C H(W)$; Fig. 1 illustrates the concept with a FC grasp and a non FC grasp in a hypothetical 2D wrench space (the actual wrench space is 6-dimensional).

\section{E. Grasp quality}

A quality measure is required to quantify the quality of a grasp; it also provides an optimization criterion for the algorithm presented later in this paper. Several grasp quality 


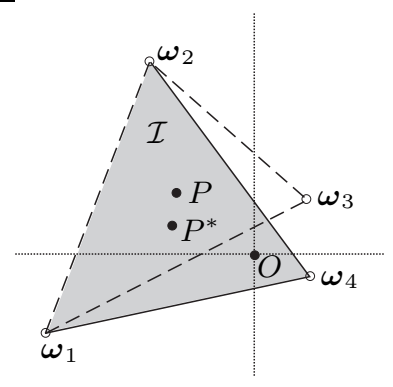

Fig. 1. Force closure test. The grasp with wrench set $W=\left\{\boldsymbol{\omega}_{1}, \boldsymbol{\omega}_{2}, \boldsymbol{\omega}_{3}\right\}$ (with $\mathrm{CH}$ represented in discontinuous lines) is non-FC. The grasp with wrench set $W^{*}=\left\{\boldsymbol{\omega}_{1}, \boldsymbol{\omega}_{2}, \boldsymbol{\omega}_{4}\right\}$ (with $C H$ represented in continuous lines) is a FC grasp.

measures have been proposed in the literature [17]; this work uses as a quality measure the largest perturbation wrench that the grasp can resist, with independence of the perturbation direction [12]. Geometrically, that quality is the radius of the largest ball centered at the origin of the wrench space and fully contained in $C H(W)$, i.e. it is the distance from the origin of the wrench space to the closest facet of $C H(W)$. This is one of the most popular grasp quality measures, and will be referred hereafter as the largest ball criterion.

\section{SYNTHESIS OF A FORCE-CLOSURE GRASP}

\section{A. The algorithm}

The synthesis of a FC grasp is based on geometric reasoning, avoiding the inclusion of an explicit FC test in the synthesis algorithm. A set $S^{1}$ of $n-1$ random points ( $n=7$ for a frictionless grasp) is selected from $\Omega$, and the convex hull $C H(W)$ of the selected points plus the origin $O$ of the wrench space is computed; the missing contact required to get a FC grasp must be found (in this sense, the approach uses the necessary and sufficient condition provided in [4] for the FC grasp when one or more contacts are missing). Let $H$ be a supporting hyperplane of $C H(W)$ containing the origin, and let $H^{+}$be the half-space defined by $H$ that contains $C H(W)$. The intersection of all of the half-spaces $\mathrm{H}^{+}$is the union of two convex sets, $\mathcal{C}_{1}$ and $\mathcal{C}_{2}$, as illustrated in Fig. 2. Let $\mathcal{C}_{1}$ be the convex set containing $C H(W)$, then, if there is at least one wrench in $\mathcal{C}_{2}$, it will provide a $\mathrm{FC}$ grasp when added to the set $S^{1}$, according to Lemma 1 . If $\mathcal{C}_{2}$ is empty, the algorithm iteratively replaces one of the wrenches in $S^{1}$ and performs another search of points in the new $\mathcal{C}_{2}$, until it contains at least one point, i.e. until it finds at least one FC grasp. The steps in the algorithm are: Algorithm 1: Search of a FC grasp

1) Generate a random set $S^{k}$ with the wrenches corresponding to $n-1$ contact points, $S^{k}=\left\{\boldsymbol{\omega}_{1}, \ldots, \boldsymbol{\omega}_{n-1}\right\}$, $k=1$.

2) Build the wrench set $W^{k}$ adding the origin $O$ of the wrench space to the set $S^{k}$.

3) Compute the convex hull $C H\left(W^{k}\right)$.

4) Use the supporting hyperplanes of $C H\left(W^{k}\right)$ containing the origin to look for the points lying in the sets $\mathcal{C}_{1}$ and $\mathcal{C}_{2}$.

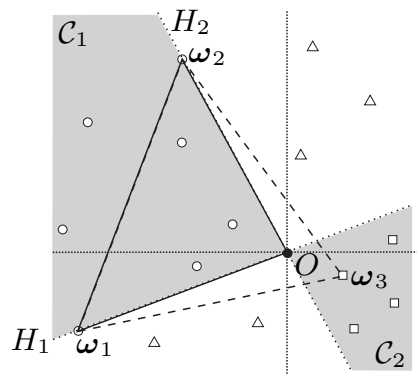

Fig. 2. Synthesis of a FC grasp. The convex hull of the wrench set $W=\left\{\boldsymbol{\omega}_{1}, \boldsymbol{\omega}_{2}, \mathbf{0}\right\}$ (in continuous lines) defines the supporting hyperplanes $H_{1}$ and $H_{2}$ that contain the origin. The convex set $\mathcal{C}_{2}$ contains 4 points (depicted as white squares), thus the algorithm provides 4 FC grasps, one of them illustrated with the convex hull in discontinuous lines.

5) If there is at least one point in $\mathcal{C}_{2}$, then the algorithm finishes and returns at least one FC grasp $G$. If $\mathcal{C}_{2}$ is empty, choose one of the wrenches in $\Omega-\mathcal{C}_{1}-\mathcal{C}_{2}$, and use it to replace the closer wrench in the set $S^{k}$, thus generating $S^{k+1}$. Go to Step 2 .

To progressively explore the search space and assure the completeness of the algorithm, the wrenches lying in $\mathcal{C}_{1}$ are labeled as explored wrenches. When $\mathcal{C}_{2}$ is empty, any combination of $n-1$ wrenches in $\mathcal{C}_{1}$ (including the wrenches in $S^{k}$ ) will not yield a FC grasp, thus all of these possible combinations are left out for subsequent searches. The algorithm keeps running and finishes when at least one FC grasp is obtained; the algorithm provides as many FC grasps as points lie in $\mathcal{C}_{2}$.

The algorithm was extended to frictional grasps. The primitive contact wrenches are used to compute the convex hull $C H(W)$, and any primitive wrench lying in $\mathcal{C}_{2}$ will provide a FC grasp when the wrenches of the corresponding grasp point are added to the set $S^{k}$. A more conservative approach may require that all of the primitive contact wrenches for a frictional grasp point lie inside $\mathcal{C}_{2}$ to consider it as valid grasp point; this would lead only to the more robust FC grasps, but may miss some other solutions.

\section{B. Comparison with previous works}

There is relatively little work concerning the synthesis of a FC grasp on discretized 3D objects. Niparnan and Sudsang [11] generate a number of 4-finger concurrent FC grasps to provide the user with a large set of grasps, so the user can choose an optimum one according to a quality measure appropriate for the particular task. The algorithm is based on the localization of regions in the 3D space where the axes of the friction cones seem to intersect. For each region, subsets of four grasp points are tested for the FC condition, by choosing an arbitrary point in the region and test if it is included in the four friction cones. The algorithm is capable of computing hundreds of FC grasps; the running times are below 3 seconds to get one FC grasp in objects described with 2000 surface points. However, they do not take into account any measure of grasp quality.

In a work closer to ours, Liu et al. [10] look for a force-closure grasp with frictional or frictionless contact 


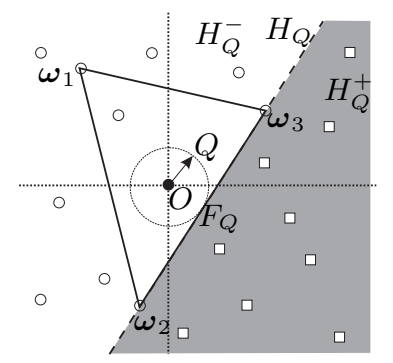

Fig. 3. Selection of the subset $\Omega_{C}^{k}$ of candidate points (depicted as white squares in the gray area) that may improve the grasp quality; in this example, $F_{Q}=\overline{\boldsymbol{\omega}_{2} \boldsymbol{\omega}_{3}}$.

points. First, an initial random grasp is chosen, and the algorithm iteratively moves the fingers to decrease the distance between the convex hull and the origin. When the procedure is trapped in a local minimum, the point set is divided into subsets by using a separating hyperplane in the wrench space, and the FC search is decomposed into subproblems, based on existence conditions for the FC grasp. The algorithm uses a FC test in each iteration; the FC test implies the solution of a linear programming problem based on the ray-shooting technique [15]. The algorithm is complete, in the sense that it finds a FC grasp if it exists in the discrete sampling of the surface, but it does not assure any optimality. The number of iterations in several examples is provided, but no information is reported on computational times.

The algorithm presented here also looks for the FC grasp in the wrench space; it is applicable to any number of frictional or frictionless fingers, and progressively covers the search space until it finds a FC grasp, or until all of the space has been covered and no FC grasp is found. It is computationally simpler than the algorithm in [10], as it does not include an explicit FC test in the algorithm; the FC condition is embedded in the search process. Moreover, the algorithm finds one or more FC grasps, depending on the number of wrenches lying in the subset $\mathcal{C}_{2}$; if there are several FC grasps, they can be classified according to a quality measure to pick the best candidate among the grasps provided by the algorithm. If a more robust grasp is required, the selected initial grasp may be optimized with the algorithm presented in the following section.

\section{SYNTHESIS OF A LOCALLY OPTIMUM GRASP}

The optimization algorithm begins with an initial FC grasp obtained, for instance, with the procedure described in the previous section. The optimization is done according to the largest ball criterion. The steps in the algorithm for the frictionless case are:

\section{Algorithm 2: Search of a locally optimum FC grasp}

1) Find an initial FC grasp, $G^{k}=\left\{\boldsymbol{\omega}_{1}, \ldots, \boldsymbol{\omega}_{7}\right\}, k=1$, using the algorithm presented in Section III, and form the corresponding wrench set $W^{k}$.

2) Determine $F_{Q}$, the facet of the convex hull $\mathrm{CH}\left(W^{k}\right)$ closest to the origin. The distance from the origin $O$ to $F_{Q}$ is the current grasp quality $Q^{k}$.

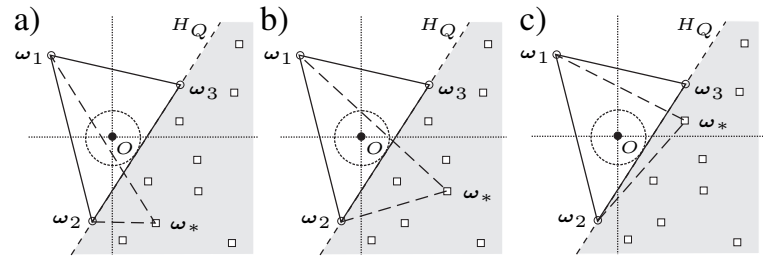

Fig. 4. Possible cases for a candidate grasp in the optimization procedure: a) Non-feasible candidate grasp, b) Discarded candidate grasp, c) Feasible candidate grasp.

3) Build the subset $\Omega_{C}^{k}$ with the candidate points that may produce an improvement in the grasp if they replace one point in $F_{Q}$. Let $H_{Q}$ be the hyperplane containing the facet $F_{Q}$. The subset $\Omega_{C}^{k}$ contains the points lying in the open half-space defined by $H_{Q}$ that does not contain the origin $O$, i.e. $H_{Q}^{+}$, as illustrated in Fig. 3 for a hypothetical two-dimensional wrench space.

4) Generate 6 candidate grasps $G_{i}^{*}, i=1, \ldots, 6$, by picking one point $\omega_{*}$ from $\Omega_{C}^{k}$ and replacing each one of the vertices defining the facet $F_{Q}$. Due to the selection procedure, all the wrenches $\omega^{*} \in \Omega_{C}^{k}$ are external points to $C H(W)$, therefore, when replacing one vertex $\boldsymbol{\omega}_{i}$ from the actual $C H$ with the candidate wrench $\boldsymbol{\omega}_{*}$, the latter will become a vertex of the new $C H$. The explicit computation of the new $\mathrm{CH}$ is not required, as its facets are constructed from the old ones replacing $\boldsymbol{\omega}_{i}$ with $\boldsymbol{\omega}_{*}$.

5) Check the candidate grasps for the FC property using Lemma 1. For the FC candidate grasps, the expected grasp quality $Q^{*}$ is computed; if for any candidate grasp $Q^{*}>Q^{k}$, then the candidate becomes the new grasp $G^{k+1}$. Fig. 4 illustrates three possible cases related to the candidate grasps; case (a) is a non-feasible grasp because it loses the FC property, case (b) is discarded because the grasp has a smaller quality than the previous one, and case (c) is a FC grasp that improves the current grasp quality, thus it becomes the grasp for the next iteration cycle. If any candidate grasp improves the quality, the algorithm goes back to Step 2. If there is no improvement in $Q^{k}$ once all the points in $\Omega_{C}^{k}$ have been considered, then a local minimum has been reached, the algorithm finishes and returns the current grasp $G$.

When frictional contacts are considered, the vertices defining the facet $F_{Q}$ are primitive contact wrenches associated to different grasp points $\boldsymbol{p}_{i}$ (Fig. 5). Therefore, the candidate grasps in Step 4 are generated by replacing all the contact points $\boldsymbol{p}_{i}$ that contribute with at least one primitive contact wrench in the facet $F_{Q}$. When a grasping point is substituted by a new one in Step 5, the explicit computation of the new convex hull is required, which increases the computational complexity of the algorithm in the frictional case.

To the best of the authors knowledge the algorithms presented in the literature for FC grasp synthesis in discretized 3D objects focus only on getting one FC grasp, regardless of whether it is (locally) optimal or not. As a consequence, no direct comparison can be established between the present and other previously published works. 


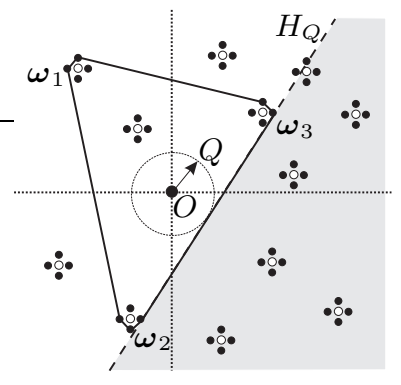

Fig. 5. Optimization procedure for frictional grasps. White circles represent normal contact wrenches, and black circles are the corresponding primitive contact wrenches. Wrenches falling in the gray zone belong to the subset $\Omega_{C}^{k}$ of candidate points that may improve the grasp quality.
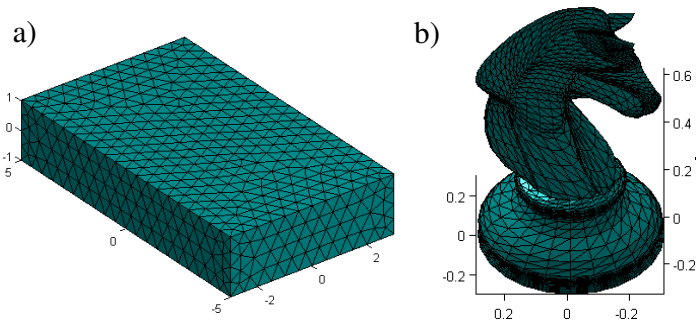

Fig. 6. Objects used in the examples: a) Parallelepiped discretized with a mesh of 1628 triangles, b) Knight discretized with 4750 triangles.

\section{EXAMPLES}

The proposed algorithms have been implemented using Matlab on a Pentium IV $3.2 \mathrm{GHz}$ computer. The performance of the approach is illustrated using the two objects shown in Fig. 6: a parallelepiped and a chess knight. The object surfaces are represented with triangular meshes. The contact points $\boldsymbol{p}_{i}$ considered on the object surface are the centroids of the triangles in the mesh, and the corresponding surface normal directions are the directions normal to the triangles.

\section{A. Example 1: frictionless grasp of a parallelepiped}

The parallelepiped is described with a mesh of 1628 triangles. This simple figure makes more difficult the search of the first FC grasp, as the initial randomized grasp may place all the fingers on just one or two faces (because there are two large faces, the probability of placing a finger on those faces is greater than on the others). Fig. 7a and 7b show the initial and locally optimal FC grasps for a particular case. The time elapsed to obtain the initial FC grasp is 0.13 seconds in 2 iterations; Algorithm 1 provides other 22 possible FC grasps. The time to get the locally optimum grasp with Algorithm 2 is 16.8 seconds in 21 iterations. Fig. 7c plots the grasp quality in the optimization phase; the quality always increases monotonically until it finds the locally optimum grasp.

The obtained locally optimum grasp depends on the initial FC grasp. In the example, the initial grasp quality is $Q_{I}=$ 0.04 , and the locally optimum gasp has a quality of $Q_{F}=$ 0.212 . The improvement factor $Q_{F} / Q_{I}$ is 5.3. However, as the improvement factor depends on the initial FC grasp, 50 a)
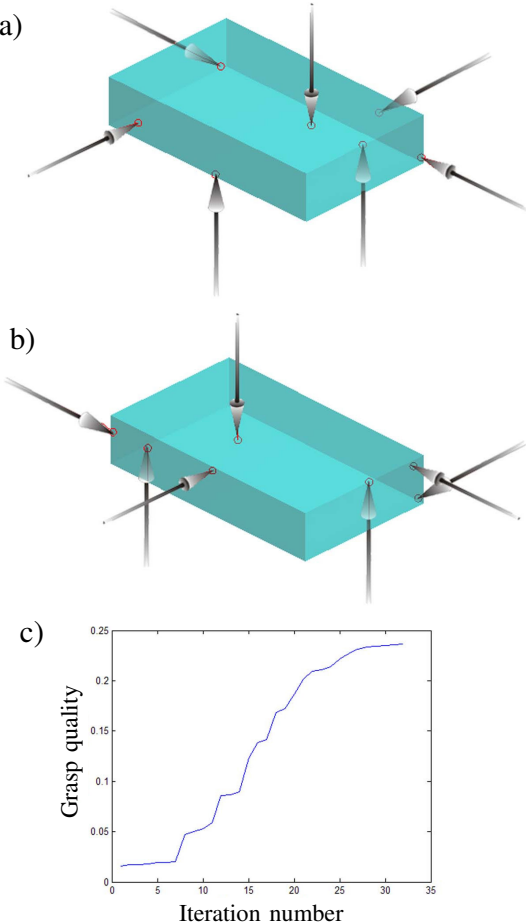

Fig. 7. FC grasp on the parallelepiped: a) Initial FC grasp, b) Locally optimum FC grasp, c) Increase in the grasp quality.

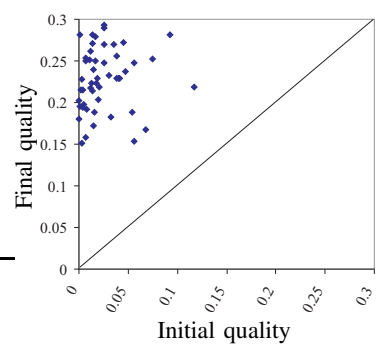

Fig. 8. Initial vs. final quality for the parallelepiped frictionless grasps.

locally optimum grasps were computed to obtain a better insight into the performance of the whole process. The correlation between the initial and final qualities is shown in Fig. 8. The average qualities give an idea of the behavior of the algorithm, they are 0.025 and 0.227 for the initial and locally optimum FC grasps, respectively; the average improvement factor is 9 .

\section{B. Example 2: frictionless grasp of a knight}

The knight is discretized with 4750 triangles (Fig. 6b). Fig. 9 shows the results for a particular case; the initial grasp is found in 0.2 seconds with no iterations (the initial random wrenches yield a FC grasp), and 49 additional FC grasps are provided. The locally optimum grasp is obtained after 35 iterations in 31.6 seconds. The grasp qualities are 0.009 and 0.058 for the initial and locally optimum FC grasps, respectively, with an improvement factor of 6.4. Fig. 10 shows the correlation between initial and final grasp qualities in 50 cases. The average quality for the initial FC grasp is 0.0052 , and 0.067 for the locally optimum grasp; the average 
a)

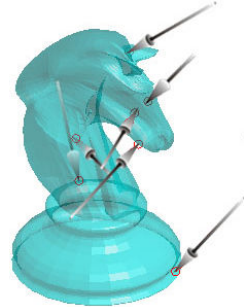

b)

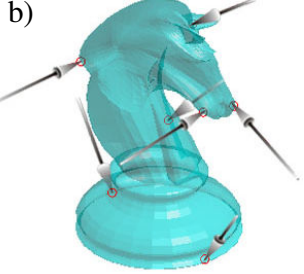

Fig. 9. Frictionless grasp on the knight: a) Initial FC grasp, b) Locally optimum FC grasp.

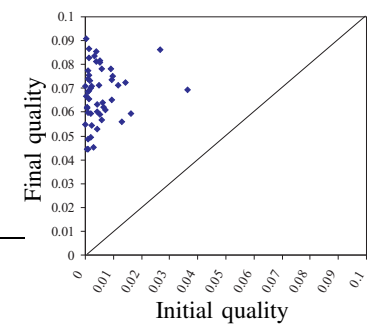

Fig. 10. Initial vs. final quality for the knight frictionless grasps.

improvement factor is 12.9 .

\section{Example 3: frictional grasp of a knight}

The frictional grasps on the knight are computed considering a friction coefficient of 0.2 and 4 fingers (the minimal number of fingers to guarantee a frictional FC grasp). The friction cones have been linearized with an 8-side polyhedral convex cone. Fig. 11 shows the results for a particular case; Algorithm 1 provides 16 FC grasps in 11 seconds and 1 iteration. The locally optimum grasp is obtained after 16 iterations in 13 minutes. Fig. 12 shows the correlation between initial and final grasp qualities for 50 initial and locally optimum grasps. The average quality for the initial FC grasp is 0.015 , and 0.04 for the locally optimum grasp. The average improvement factor is 2.8 .

\section{CONCLUSions}

This paper proposes a geometrical approach to obtain at least one force-closure (FC) grasp in 3D discretized objects, with frictional or frictionless contacts and with any number of fingers. The presented algorithm embeds the FC condition in the procedure to avoid an explicit FC test in each iteration. The initial FC grasp may be improved with an oriented search procedure, optimizing the grasp quality according to the largest ball criterion. The algorithms were implemented and the execution results (as the examples shown in the paper) illustrate the relevance and efficiency of the approach. Future work includes the determination of independent contact regions for $3 \mathrm{D}$ discretized objects, such that placing a finger in each contact region assures a force-closure grasp on the object, independently of the exact position of the contact points, and providing robustness in front of finger positioning errors in grasp and fixturing applications.

\section{REFERENCES}

[1] A. Bicchi, "On the closure properties of robotic grasping," Int. J. Robotics Research, vol. 14, no. 4, pp. 319-344, 1995.

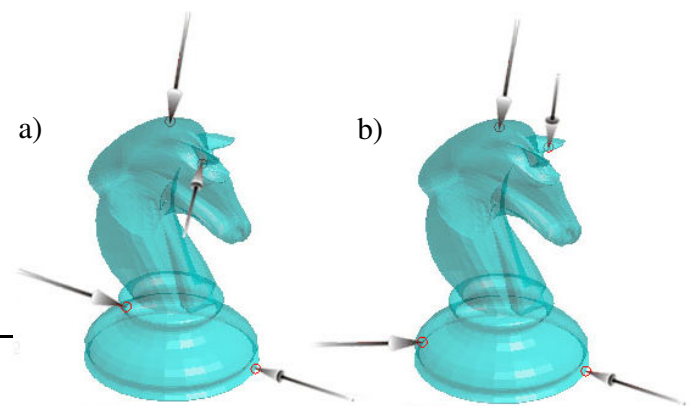

Fig. 11. Frictional grasp on the knight: a) Initial FC grasp, b) Locally optimum FC grasp.

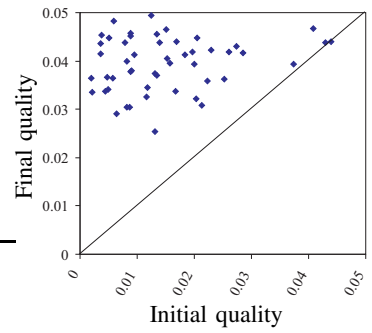

Fig. 12. Initial vs. final quality for the knight frictional grasps.

[2] Y. Liu, "Computing n-finger form-closure grasps on polygonal objects," Int. J. Robotics Research, vol. 19, no. 2, pp. 149-158, 2000.

[3] J. Cornellà and R. Suárez, "On computing form-closure grasps/fixtures for non-polygonal objects," in Proc. IEEE Int. Symp. Assembly and Task Planning, ISATP 2005, 2005, pp. 138-143.

[4] D. Ding, Y. Liu, and S. Wang, "Computation of 3-D form-closure grasps," IEEE Trans. Robotics and Automation, vol. 17, no. 4, pp. 515-522, 2001

[5] X. Zhu and J. Wang, "Synthesis of force-closure grasps on 3-D objects based on the $\mathrm{Q}$ distance," IEEE Trans. Robotics and Automation, vol. 19 , no. 4, pp. 669-679, 2003.

[6] R. Campbell and P. Flynn, "A survey of free-form object representation and recognition techniques," Computer Vision and Image Understanding, vol. 81, pp. 166-210, 2001.

[7] C. Borst, M. Fischer, and G. Hirzinger, "Grasping the dice by dicing the grasp," in Proc. IEEE/RSJ IROS 2003, 2003, pp. 3692-3697.

[8] M. Wang, "An optimum design for 3-D fixture synthesis in a point set domain," IEEE Trans. Robotics and Automation, vol. 16, no. 6, pp. 839-846, 2000.

[9] D. Ding, Y. Liu, and M. Wang, "On computing inmobilizing grasps of 3-D curved objects," in Proc. IEEE Int. Symp. on Computational Intelligence in Robotics and Automation, 2001, pp. 11-16.

[10] Y. Liu, M. Lam, and D. Ding, "A complete and efficient algorithm for searching 3-D form closure grasps in the discrete domain," IEEE Trans. Robotics, vol. 20, no. 5, pp. 805-816, 2004.

[11] N. Niparnan and A. Sudsang, "Fast computation of 4-fingered forceclosure grasps from surface points," in Proc. IEEE IROS 2004, 2004, pp. 3692-3697.

[12] C. Ferrari and J. Canny, "Planning optimal grasps," in Proc. IEEE ICRA 1992, 1992, pp. 2290-2295.

[13] B. Mishra, J. Schwartz, and M. Sharir, "On the existence and synthesis of multifinger positive grips," Algorithmica, vol. 2, no. 4, pp. 541-558, 1987.

[14] R. Murray, Z. Li, and S. Sastry, A Mathematical Introduction to Robotic Manipulation. Boca Ratón, Florida: CRC Press, 1994.

[15] Y. Liu, "Qualitative test and force optimization of 3-D frictional formclosure grasps using linear programming," IEEE Trans. Robotics and Automation, vol. 15, no. 1, pp. 163-173, 1999.

[16] E. Gilbert, D. Johnson, and S. Keerthi, "A fast procedure for computing the distance between complex objects in three-dimensional space," IEEE J. Robotics and Automation, vol. 4, no. 2, pp. 193-203, 1988

[17] K. Shimoga, "Robot grasp synthesis algorithms: A survey," Int. J. Robotics Research, vol. 15, no. 3, pp. 230-266, 1996. 